\title{
Diversidad de Psocoptera (Insecta: Psocodea) en el Parque Nacional Natural Gorgona (Océano Pacífico, Colombia)
}

\author{
Fabio Sarria-S. ${ }^{1 *}$, Alfonso Neri García-Aldrete ${ }^{2} \&$ Ranulfo González O. ${ }^{1}$ \\ 1. Facultad de Ciencias Naturales y Exactas, Universidad del Valle, Calle 13, 100-00, Fax 3393243, tel. 57-2-3212100 \\ ext.3137, Santiago de Cali, Colombia; fabsarria@gmail.com; ranulfo.gonzalez@correounivalle.edu.co \\ 2. Departamento de Zoología, Instituto de Biología, Universidad Nacional Autónoma de México. Apartado Postal \\ 70-153, 04510 México, D. F., MÉXICO; anga@ibunam2.ibiologia.unam.mx
}

Recibido 18-X-2013. Corregido 20-XI-2013. Aceptado 19-XII-2013.

\begin{abstract}
Diversity of Psocoptera (Insecta: Psocodea) at the National Natural Park Gorgona (Pacific Ocean, Colombia). Psocopterans are insects that have been recorded from all zoogeographic regions but have more species in the tropics. We studied their diversity of in four habitats (Beaches, Village, Trails and Arboreal strata) at the Gorgona National Natural Park, Colombia, from samples collected during November 2009 and October 2010. We added the arboreal Psocoptera collected by canopy fogging in November 2007. The specimens collected were identified to genus and/or to species or morphospecies level. Comparisons were made of the richness and diversity among sites. Although there was no significant difference in the diversity estimated by the Shannon-Wiener index, high species richness and abundance were recorded for the Beaches and Village habitats. The psocid community of Gorgona occurs mainly where vegetation cover is low. Rev. Biol. Trop. 62 (Suppl. 1): 257-264. Epub 2014 February 01.
\end{abstract}

Keys words: Neotropical Psocoptera, humid tropical forest, vegetation cover.

Psocoptera es un orden de amplia distribución mundial, que a partir de estudios en áreas tropicales ha incrementado notablemente el número de especies descritas. Se encuentran en todas las regiones zoogeográficas del mundo, presentando mayor riqueza de especies en los trópicos, como lo indican los valores de diversidad compilados por García-Aldrete (1988). Se encuentran en troncos y follaje de árboles, arbustos y herbáceas, en hojarasca del suelo, en superficies de rocas, y en ambientes humanos en paredes, libros y graneros, ocasionalmente pueden estar asociados con granos almacenados (Mockford, 1993).

Los psócidos hacen parte del plancton aéreo, siendo de los primeros artrópodos en colonizar la vegetación (Simberloff \& Wilson, 1969) y constituyen una fracción significativa de la biomasa en bosques templados (Thornton, 1985), por lo cual se infiere que son organismos clave de la red trófica de muchos ecosistemas. Se han registrado 26 taxones de depredadores de estos organismos, siendo los más frecuentes arañas, ácaros, opiliones, larvas de neurópteros, algunos coccinélidos y varias especies de aves pequeñas; se ha registrado que los huevos son parasitados por avispas Mymaridae y las ninfas por Braconidae (Euphoriella) (Broadhead, 1958; Turner \& Cheke, 1973; Turner, 1984).

Lo anterior, sumado a los cambios y actualizaciones de la clasificación del orden plantea la necesidad de realizar un estudio preliminar del orden en Colombia, con el fin de ampliar al conocimiento de este grupo para el país. En este contexto, el Parque Nacional Natural Gorgona, por su ubicación geográfica y variedad de hábitats, producto de la confluencia de varios fenómenos climáticos, atmosféricos, marinos, topográficos y humanos (principalmente durante el funcionamiento del penal entre 1960 y 
1985), lo convierten en un sitio ideal para el estudio de la diversidad y distribución de los psócidos del Pacífico colombiano.

Aprovechando el escenario de investigación que ofrece el PNN Gorgona, se han estudiado los artrópodos del suelo, de cuerpos de agua y de estratos arbóreos (Torres, Pinilla \& Chamorro, 1998; Cala, 1990; Andrade, Cadena, Gómez, Uribe \& Benavidez, 1991; Zapata, Beltrán, Collazos \& Von Prahl, 1991; Manzano, Nieser \& Caicedo, 1995; Longo, Gómez, Blanco \& Zamora, 2008; Pimienta, Montealegre, Chacón de Ulloa, Gonzalez \& Mejía, 2008; Gómez, 2009). En cuanto a Insecta, se han llevado a cabo investigaciones sobre Formicidae (Ulloa-Chacón, 1990; Baena, 1992), Lepidoptera (Andrade, 1991), Diptera (Montoya \& Baena, 1995) y Orthoptera (Sarria, Montealegre, Mason \& Pimienta, 2008). Aunque a partir de una revisión preliminar de la colección de artrópodos del PNN Gorgona, depositada en el Museo de Entomología de la Universidad del Valle (MUSENUV), realizada por Andrew, Mason \& Montealegre en 2007, se logró establecer la presencia de este grupo en la isla, hasta la fecha no se han realizado investigaciones en esta localidad en donde se relacione a Psocoptera con aspectos ecológicos o taxonómicos (UAESPNN, 2008).

El estudio de Psocoptera en la isla Gorgona se realizó con el objetivo de establecer la diversidad de este grupo en el parque, a través de la comparación de cuatro zonas: playas, asentamientos humanos, estratos arbóreos y senderos a través del bosque primario y secundario, convirtiéndose este estudio en el primero en aportar información técnica sobre este grupo para el PNN Gorgona.

\section{MATERIALES Y MÉTODOS}

Área de estudio: El Parque Nacional Natural Gorgona incluye las islas de Gorgona y Gorgonilla. Se encuentra ubicado al sur del Pacífico colombiano (2॰58'34" N - 78 12 '51" W), y es jurisdicción del municipio de Guapi, en el departamento de Cauca. El área terrestre es de $13.8 \mathrm{Km}^{2}$ y su elevación varía desde 0 hasta 338msnm en el cerro la Trinidad (Chamorro, 1990). Climáticamente, la isla se clasifica como súper húmeda sin deficiencia de agua y mega termal con baja concentración de calor en el período de menor pluviosidad (Rangel \& Rudas, 1990).

En el área terrestre, la cobertura vegetal puede diferenciarse entre vegetación intervenida y natural. La primera es el resultado de la época del penal, durante la cual se usó el sector oriental de la isla para el establecimiento de la infraestructura carcelaria, constituida por la prisión (cuatro patios), aproximadamente 30 casas donde se alojaba el personal oficial, un comedor, un muelle y una zona utilizada como pista de aterrizaje para pequeñas aeronaves; además se hizo un consumo masivo de madera obtenida de los bosque cercanos. En cuanto a la vegetación natural, se agrupa en tres asociaciones: Ossaeo sessilifoliae-Anaxagoretum phaeocarpae, Malpighio glabrae-Cespedesietum macrophyllae y Cassipoureo ellipticae-Ryanetum speciosae y dos comunidades: Perebea xanthochyma-Cosmibuena macrocarpa y Symphonia globulifera-Hyeronima oblonga-Terminalia amazonia (UAESPNN, 2005).

Muestreos: Se llevaron a cabo durante dos campañas, una realizada en noviembre de 2009 (18h/hombre) y la otra en octubre de 2010 (12h/hombre). Se realizaron 14 eventos de recolecta diurnos en corteza de árboles (directa) y vegetación (ramas vivas y muertas), en este último se realizó golpeo sobre una bandeja recolectora de $0.12 \mathrm{~m}^{2}$. En ambos casos se utilizó un aspirador bucal para recolectar los especímenes observados, tanto en la corteza como en la bandeja recolectora. Se usó el tiempo como medida del esfuerzo de muestreo para asegurar la comparación de las comunidades entre los biotopos; de tal modo que una unidad de muestreo correspondía a dos horas de tiempo ininterrumpido en cada evento de recolecta.

Para comparar la diversidad de psócidos entre las diferentes zonas, se establecieron $a$ priori cuatro grupos que se designaron como: "Playas", "Senderos", "Poblado" y "Arbóreo" ( 5 a $20 \mathrm{~m}$ de altura desde el suelo). El criterio 
de agrupamiento consistió en el tipo de bosque y cobertura vegetal promedio, que fue medida en campo en los sitios muestreados con un densiómetro esférico a lo largo de la zona de recolecta con un intervalo de $10 \mathrm{~m}$ entre puntos de medición (Cuadro 1).

CUADRO 1

Cobertura vegetal promedio para los sitios estudiados en el PNN Gorgona

TABLE 1

Mean canopy coverage for beaches, village, trails and forest trails at the NNP Gorgona

\begin{tabular}{lcc}
\multicolumn{1}{c}{ Sitio } & Cobertura vegetal promedio (\%) & $\mathrm{n}$ \\
\hline Playas & 52.47 & 14 \\
Poblado & 56.20 & 19 \\
Senderos & 84.25 & 28 \\
Senderos Bosque & 93.93 & 21 \\
\hline
\end{tabular}

Las recolectas correspondientes al hábitat "Playas", se realizaron en las playas Blanca, Palmeras y de Gorgonilla. En estas se distinguen zonas de cultivo de Cocos nucifera L., Citrus medica L., y árboles frutales como Psidium guajaba L. y Persea americana Mill., también se encuentran comunidades de plantas pioneras, compuestas por Canavalia maritima (Aubl.) Thouars y Vigna peduncularis (Kunth) Fawc. \& Rendle.

Para los muestreos en el hábitat "Senderos", bordes de bosque y sotobosque, se recorrieron los trayectos ya establecidos en el parque con fines recreativos y de monitoreo, tales como: (a) Poblado-Playa Yundigua, con una longitud de 2282m; (b) La Chonta, que es un circuito que conecta al Poblado con la microcentral hidroeléctrica y el centro de Interpretación; tiene una longitud de $1080 \mathrm{~m}$; (c) Poblado-Playa Palmeras, con $4290.9 \mathrm{~m}$ de longitud. La cobertura vegetal de estos sitios es de tipo intervenida en etapa de sucesión, en la mayoría de los casos, las especies dominantes en el estrato arbóreo son: Vismia baccifera (L.) Triana \& Planch., Cecropia garciae Standl., y Cespedesia macrophylla Seem., en el estrato herbáceo aparecen Triolena spicata (Triana) L.O. Williams, Tara sp., Tetrochidium gorgonae Croizat y Wedelia triloba (L.) Hitchc. (UAESPNN, 2005).

Adicionalmente, se llevaron a cabo dos eventos de recolecta al interior del bosque, con ascensos a los cerros de La Trinidad y el Mirador, cuya cobertura vegetal fitosociológicamente corresponde a la alianza Cespedesia-Symphonia globuliferae, que comprende vegetación de tipo selvático, con elementos de $35 \mathrm{~m}$ de altura. Entre las especies características del dosel y de la bóveda de la fitocenosis se destacan: C. macrophylla, Symphonia globulifera L. F., Ardisia opaca Lundell, Perebea xanthochyma H. Karst., Eschweilera pittieri R. Knuth y Casearia sylvestris Sw. (UAESPNN, 2005).

Para el hábitat "Poblado", y considerando que está constituido por aproximadamente 30 cabañas, un restaurante y un museo pequeño, los muestras se tomaron de diversas estructuras elaboradas con madera (muebles, puertas, ventanas y objetos decorativos), techos con cobertura de hojas de palma y plantas ornamentales. Este sector tiene un área aproximada de tres hectáreas, incluyendo la playa, ubicada entre el antiguo muelle y la zona nororiental del mismo, y concentra la infraestructura administrativa del parque, turística y de investigación (UAESPNN, 2005).

Para el hábitat "Arbóreos", se revisó la colección de artrópodos arbóreos del Parque Nacional Natural Isla Gorgona, depositada en el Museo de Entomología de la Universidad del Valle (MUSENUV); ejemplares obtenidos del muestreo realizado por Andrew, Mason \& Montealegre en 2007, quienes muestrearon 16 árboles (diez en un bosque secundario y seis en un bosque primario) del sector oriental de la isla, mediante la técnica de nebulización del dosel.

Los especímenes recolectados se preservaron en etanol al $80 \%$, y posteriormente, en el laboratorio del grupo de investigaciones entomológicas (GIE) de la Universidad del Valle, se identificaron y cuantificaron. En algunos casos, se realizaron preparaciones en placas de vidrio para la identificación genérica y/o específica, de las alas anteriores, cabeza y genitales, siguiendo el método descrito en González-Obando, García-Aldrete \& Carrejo 
(2011). Para la identificación se usaron las claves presentadas en Smithers (1990) y Mockford (1993).

Análisis estadístico: Con base en el número de especies y la abundancia relativa, se estimó la riqueza de especies (S) y el índice de diversidad de Shannon-Wiener (H') (Magurran, 1988), para cada sitio en el programa Past ${ }^{\circledR} 2.0$ (Hammer, Harper \& Ryan, 2001). Posteriormente, se compararon los valores obtenidos para cada hábitat mediante un análisis de varianza de una vía de medidas repetidas, donde el factor de estudio fue el tipo de hábitat. Previamente se evaluaron los supuestos de normalidad y homogeneidad de varianza mediante una prueba de Shapiro-Wilk y de Bartlett, respectivamente. Para el cálculo de estos análisis se utilizó el programa STATISTICA ${ }^{\circledR} 8.0$ (Statsoft 2007).

La eficiencia del esfuerzo de muestreo fue evaluada a partir de los estimadores no paramétrico de la riqueza que se basan en la abundancia, tales como Chao 1 (Chao, 1984), ACE (Chazdon, Colwell, Denslow \& Guariguata, 1998), Jacknife 1 y 2 (Palmer, 1990). Las estimaciones se realizaron con el programa EstimateS® 8.0 de Colwell (2009).

\section{RESULTADOS}

Se recolectaron 1237 ejemplares, identificados en 62 morfoespecies (Cuadro 2). Las especies más abundantes fueron Echmepteyx madagascariensis Kolbe, con 236 ejemplares, E. falco Badonnel con 196 ejemplares y Heterocaecilius sp.1 con 119 ejemplares. A pesar de que estuvieron presentes en los cuatro biotopos, su mayor abundancia se registró en las "Playas" y el "Poblado", con 392 y 89 individuos respectivamente (Cuadro 1).

En el Cuadro 3 se presenta la riqueza de especies promedio y acumulada para cada hábitat y los valores estimados por el índice de Shannon-Wiener para los mismos. Adicionalmente, al comparar los hábitats evaluados, se encontró que el hábitat "Playas" aportó el $53 \%$ de la abundancia total de las muestras y en contraste el hábitat "Senderos" presentó el porcentaje más bajo, con el $9 \%$ de la abundancia relativa.

El análisis de varianza para las comparaciones de la riqueza específica promedio y los valores estimados por el índice de ShannonWiener entre hábitats, mostró que las "Playas", con 16.3 especies en promedio (Cuadro 3), presentan diferencia significativa $(p=0.002)$; para la riqueza y para el índice de ShannonWiener no hubo diferencia significativa entre los hábitats $(\mathrm{p}=0.67)$.

El esfuerzo de muestreo para la isla de 24 eventos de recolecta presentó una efíciencia promedio del $67.7 \%$, lo cual indica que con un número mayor de recolectas se podría llegar a obtener una mejor representación de la riqueza de Psocoptera para el PNN Gorgona (Cuadro 4).

\section{DISCUSIÓN}

Mockford (1993), señala que especies como Echmepteyx madagascariensis Kolbe, E. falco Badonnel, que presentaron una alta abundancia relativa (Cuadro 2), son especies ampliamente distribuidas en el trópico, asociadas a hojas secas de especies de las familias Arecaceae y Musaceae. Son consideradas especies pioneras en los procesos de sucesión temprana en algunas partes del Pacífico (Thornton, New \& Vaughan, 1988), lo cual puede explicar su presencia y abundancia en la isla. A pesar de que estuvieron presentes en los cuatro hábitats estudiados, particularmente para los hábitats "Playas" y "Poblado", se registró la mayor riqueza y abundancia asociada a plantas de C. nucifera y de Croton sp., lo cual coincide con los resultados obtenidos por Thornton et al. (1988), en el archipiélago de Krakatoa, Indonesia, donde especies de la familia Lepidopsocidae fueron las más abundantes en este tipo de vegetación. En contraste, solo se recolectaron uno o dos ejemplares de Proentomum personatum Badonnel, Nepticulomima hoesemanni Enderlein y varios de Mesepipsocus sp., en el "Poblado", asociados a estructuras de madera 
CUADRO 2

Especies de Psocoptera encontradas en cuatro habitats del PNN Gorgona.

TABLE 2

Psocoptera species found in four habitats of the NNP Gorgona.

\begin{tabular}{|c|c|c|c|c|c|c|c|c|c|c|c|}
\hline Especie & Py & $\mathrm{Pb}$ & $\mathrm{Ar}$ & $\mathrm{Sd}$ & $\mathrm{n}$ & Especie & Py & $\mathrm{Pb}$ & $\mathrm{Ar}$ & $\mathrm{Sd}$ & $\mathrm{n}$ \\
\hline Seopsocus sp. & & & 2 & & 2 & Lichenomima sp. & 2 & & & & 2 \\
\hline Archipsocus sp. 1 & 2 & 1 & 1 & 1 & 5 & Tapinella sp. & & & & 3 & 3 \\
\hline Archipsocus sp. 2 & 1 & & 8 & & 9 & Peripsocus sp. 1 & 2 & 7 & 5 & & 14 \\
\hline Archipsocus sp. 3 & 10 & 9 & & 1 & 20 & Peripsocus sp. 2 & & 1 & & & 1 \\
\hline Pararchipsocus sp. & 30 & & & & 30 & Peripsocus sp. 3 & & & 1 & & 1 \\
\hline Notiopsocus sp. & 1 & & & & 1 & Gen. ca. Kaestneriella sp. & 1 & 2 & & & 3 \\
\hline Valenzuela sp. 1 & & & 7 & & 7 & Aaroniella sp. & 3 & & 9 & & 12 \\
\hline Valenzuela sp. 2 & 7 & 51 & & 18 & 76 & Heterocaecilius sp. 1 & 106 & 9 & 1 & 3 & 119 \\
\hline Valenzuela sp. 3 & & 13 & 11 & & 24 & Heterocaecilius sp. 2 & & 3 & 1 & & 4 \\
\hline Valenzuela sp. 4 & & 8 & 2 & 1 & 11 & Pseudocaecilius citricola (Ashmead) & 6 & 1 & 4 & & 11 \\
\hline Valenzuela sp. 5 & 1 & & & & 1 & Scytopsocus sp. & 13 & & 24 & & 37 \\
\hline Cladiopsocus sp. & & & 1 & & 1 & Blaste sp. & 3 & & 5 & & 8 \\
\hline Dolabellopsocus sp. & & & 2 & & 2 & Blastopsocus sp. 1 & 12 & & 5 & & 17 \\
\hline Ectopsocus sp. 1 & 20 & & & 3 & 23 & Blastopsocus sp. 2 & & & 7 & & 7 \\
\hline Ectopsocus sp. 2 & 73 & & & & 73 & Blastopsocus sp. 3 & & & 3 & & 3 \\
\hline Ectopsocus sp. 3 & 24 & 1 & & & 25 & Blastopsocus sp. 4 & 6 & 8 & & & 14 \\
\hline Ectopsocus sp. 4 & 1 & & & & 1 & Indiopsocus sp. 1 & & 1 & 5 & & 6 \\
\hline Ectopsocus sp. 5 & 5 & 4 & & 12 & 21 & Indiopsocus sp. 2 & 2 & & & 15 & 17 \\
\hline Compsocus elegans (Banks) & & 1 & 1 & & 2 & Indiopsocus sp. 3 & & & 1 & & 1 \\
\hline Goja sp. & & & & 4 & 4 & Species nov. & & & 3 & & 3 \\
\hline Mesepipsocus sp. & & 19 & & & 19 & Psococerastis sp. 1 & & & & 5 & 5 \\
\hline Hemipsocus africanus Enderlein & 26 & & & & 26 & Psococerastis sp. 2 & & & 5 & & 5 \\
\hline Lachesilla sp. 1 & & & & 3 & 3 & Psococerastis sp. 3 & & & 1 & & 1 \\
\hline Lachesilla sp. 2 & & & & 3 & 3 & Psococerastis sp. 4 & & & & 5 & 5 \\
\hline Echmepteryx falco (Badonnel) & 59 & 76 & 30 & 31 & 196 & Ptycta sp. 1 & & & 1 & & 1 \\
\hline E. madagascariensis (Kolbe) & 227 & 4 & & 5 & 236 & Ptycta sp. 2 & & & 1 & & 1 \\
\hline Lepidopsocus pretiosus (Banks) & 10 & 3 & & 1 & 14 & Thyrsopsocus sp. 1 & & & 2 & & 2 \\
\hline Proentomum personatum (Badonnel) & & & & 2 & 2 & Thyrsopsocus sp. 2 & & & & 1 & 1 \\
\hline Nepticulomima hoesemanni (Enderlein) & & 60 & & & 60 & Trichadenotecnum sp. & & & 1 & & 1 \\
\hline Belaphotroctes sp. & & & & 13 & 13 & Graphopsocus cruciatus (Linneo) & & & 4 & & 4 \\
\hline Myopsocus sp. & & & 15 & & 15 & Lepolepis sp. & 1 & & & 2 & 3 \\
\hline
\end{tabular}

$\mathrm{Py}=$ Beaches, $\mathrm{Pb}=$ Village, $\mathrm{Ar}=$ Arboreous, $\mathrm{Sd}=$ Trails.

(muebles, techos, puertas y ventanas) de edificaciones humanas.

En los senderos, la observación y recolecta de especímenes estuvo determinada por la cobertura vegetal. En los trayectos a los cerros de El Mirador y La Trinidad, no se encontraron psócopteros. En los otros senderos, como $\mathrm{La}$ Chonta, Faro Gorgonilla y el de Playa Blanca, los sitios donde se efectuaron eventos de recolecta, fueron áreas que presentaban alguna perturbación del dosel (claros por caída de árboles) o que por la topografía del terreno la cobertura vegetal disminuía (Cuadro 5).

En los estratos arbóreos (alturas entre 5 y $20 \mathrm{~m}$ desde el suelo), a excepción de tres puntos, los sitios muestreados formaban parte de una zona en recuperación, caracterizada por una baja cobertura vegetal, que proporcionaba condiciones microclimaticas que posiblemente favorecen el establecimiento de microepífitas y 
CUADRO 3

Abundancia relativa, riqueza de especies (S) y valores del índice Shannon-Wiener para cuatro sitios estudiados en el PNN Gorgona.

TABLE 3

Relative abundance, species richness (S), and Shannon-Wiener index ( $\left.\mathrm{H}^{\prime}\right)$ values for four sites studied at the NNP Gorgona.

\begin{tabular}{lccccc}
\multicolumn{1}{r}{ Sito } & Abundancia & Abundancia relativa (\%) & S: Promedio & S:Acumulada & H' $^{\prime}$ \\
Playas & 654 & 53.0 & 16.3 & 29.0 & 1.96 \\
Poblado & 282 & 24.0 & 10.0 & 21.0 & 1.48 \\
Senderos & 169 & 9.0 & 6.2 & 21.0 & 1.44 \\
Arbóreos & 132 & 14.0 & 6.5 & 35.0 & 1.52 \\
\hline
\end{tabular}

CUADRO 4

Valor pomedio de los Estimadores no parametricos de la riqueza para el PNN Gorgona

TABLE 4

Mean values of the non-parametric species estimators for the NNP Gorgona

\begin{tabular}{lcc} 
Estimador & $\begin{array}{c}\text { Riqueza } \\
\text { (Promedio) }\end{array}$ & $\begin{array}{c}\text { Eficiencia } \\
\text { del muestreo }\end{array}$ \\
Chao 1 & 87.1 & $71.0 \%$ \\
ACE & 84.4 & $73.5 \%$ \\
Jack 1 & 93.9 & $66.0 \%$ \\
Jack 2 & 102.8 & $60.0 \%$ \\
\hline
\end{tabular}

CUADRO 5

Cobertura promedio de los sitios muestreados para el hábitat "Senderos" en el PNN Gorgona

TABLE 5

Mean canopy coverage for trails and forest trails at the NNP Gorgona

\begin{tabular}{lccc}
\multicolumn{1}{c}{ Sendero } & Riqueza & Abundancia & $\begin{array}{c}\text { Cobertura } \\
\text { vegetal (\%) }\end{array}$ \\
Playa Blanca & 8 & 38 & 82.6 \\
La Chonta & 15 & 61 & 79.6 \\
Faro Gorgonilla & 4 & 12 & 72.3 \\
Playa Yundigua & 0 & 0 & 90.0 \\
La Trinidad & 0 & 0 & 96.0 \\
El Mirador & 0 & 0 & 93.0 \\
\hline
\end{tabular}

psócidos. En el bosque primario se realizaron recolectas en sitios con características similares a las del borde de bosque, entendido este como un ecotono entre el estrato arbustivo $(1.5 \mathrm{a} 6 \mathrm{~m})$ y el dosel (15 a 20m), donde se suele registrar un aumento en la temperatura, luz solar y disminución de la humedad (Foggo, Ozanne, Spleight \& Hambler, 2001).

En cuanto al esfuerzo de muestreo, los estimadores de la riqueza de especies indican que hay una deficiencia promedio del 33\% de la riqueza esperada, lo que se podría atribuir a una baja intensidad de recolectas en un sector del occidente de la isla considerado prístino (UAESPNN, 2005). Por otra parte, de acuerdo a la clasificación ecológica de Mockford (1993), la hojarasca que se acumula en el suelo de los bosques, es otro de los sustratos aprovechados por los psócidos, pero por condiciones de logística, no fue muestreada adecuadamente y se eliminó del análisis; sin embargo, en ella se encontraron dos especies adicionales.

A partir de este estudio se establece que la riqueza y diversidad de Psocoptera en el PNN Gorgona está representada por 62 especies, de las cuales dos tienen una alta abundancia de individuos y una amplia distribución en la isla. Al no encontrar diferencias estadísticas significativa entre los valores obtenidos por el índice de diversidad de Shannon-Wiener entre cada hábitat, se podría interpretar que la diversidad de los psócidos entre estos es algo similar y no es afectada por el tipo de hábitat, a pesar de haber observado una alta riqueza y abundancia de individuos en el hábitat "Playas".

Finalmente, teniendo en cuenta que los psócidos se alimentan principalmente de líquenes, hongos o algas (Thornton, 1985) y la distribución y densidad de estas microepífitas está determinada por la irradiación de la luz solar, temperatura y humedad relativa (Brodo, 
1973; Hamada, Miyawaki \& Yamada, 1995), los resultados obtenidos indican que en la isla, la distribución de los psócopteros puede estar determinada por características del hábitat, principalmente una baja cobertura vegetal, que favorece el que haya condiciones ambientales adecuadas para el establecimiento de comunidades de microepífitas, pero estudios de la relación entre micro epífitas (principalmente líquenes) y factores microclimáticos determinados por la cobertura vegetal, son indispensables para corroborar estas observaciones en el PNN Gorgona.

\section{AGRADECIMIENTOS}

FSS y RGO agradecen al Departamento de Biología, Facultad de Ciencias Naturales y Exactas, Universidad del Valle, por el apoyo a esta investigación. ANGA agradece al Instituto de Biología, Universidad Nacional Autónoma de México, el constante apoyo a su investigación. Agradecemos a Andrew Mason y a Fernando Montealegre, por permitirnos revisar el material recolectado por ellos durante el desarrollo de su proyecto en el PNN Gorgona (permiso No. DTSO-G-31); a la Administración y personal del PNN Gorgona, y a Alan Giraldo, líder del proyecto "Evaluación actual de los objetos de conservación faunísticos en la Isla Gorgona: una aproximación holística a la valoración ecológica del PNN Gorgona" (permiso No. PIBD-DTSO 011-10). Agradecemos también a Julián Alexander Mendivil, Diana Marcela Torres Domínguez y Stephany Valdés-R., su invaluable ayuda y soporte logístico durante el trabajo de campo en el sitio de estudio. Gracias también a los evaluadores anónimos por sus comentarios y sugerencias en versiones anteriores de este manuscrito.

\section{RESUMEN}

El presente trabajo tuvo como objetivo establecer la diversidad de Psocoptera en cuatro hábitats (Playas, Poblado, Senderos y Estratos arbóreos) del Parque Nacional Natural Gorgona, Colombia. Se realizaron dos campañas de recolecta durante los meses de noviembre de 2009 y octubre de 2010, con un total de 30 horas/hombre. Se revisó además el material de artrópodos arbóreos del muestreo realizado en el parque en noviembre de 2007, depositado en el Museo de Entomología de la Universidad del Valle (MUSENUV). Los especímenes recolectados en los cuatro sitios, se identificaron como mínimo a nivel de género, y posteriormente se cuantificaron para comparar la riqueza específica y diversidad entre los sitios. Aunque no hubo diferencia significativa en la diversidad estimada por el índice de Shannon-Wiener, se registró una alta riqueza de especies y abundancia para los hábitats "Playas" y "Poblado". Se observó una tendencia de los Psocoptera del PNN Gorgona a ocupar espacios donde la cubierta vegetal es baja.

Palabras clave: Psócidos neotropicales, bosque húmedo tropical, cobertura vegetal.

\section{REFERENCIAS}

Andrade, G. (1991). Inventario de mariposas (Lepidoptera: Rhopalocera). In J. Aguirre \& O. Rangel (Eds.), Biota $y$ Ecosistemas de Gorgona (pp. 252-254). Fondo FEN. Bogotá, Colombia.

Andrade, G., Cadena, A., Gómez, M., Uribe, J. \& Benavidez, P. (1991). Artropofauna asociada al suelo. In J. Aguirre \& O. Rangel (Eds.), Biota y Ecosistemas de Gorgona (pp. 41-106). Fondo FEN. Bogotá, Colombia.

Baena, M. (1992). Relaciones biogeográficas de las hormigas de la Isla Gorgona (Trabajo de pregrado). Universidad del Valle, Cali, Colombia.

Broadhead, E. (1958). The psocid fauna of larch trees in northern England: an ecological study of mixed species populations exploiting a common resource. Journal of Animal Ecology, 27: 217-263.

Brodo, I. M. (1973). Substrate ecology. In V. Ahmadjian, M. E. Hale Jr. (Eds.), The Lichens (pp. 401-441). Academic Press, Nueva York.

Cala, P. (1990). Biodiversidad en aguas dulces de la isla. In J. Aguirre \& O. Rangel (Eds.), Biota y ecosistemas de Gorgona (pp. 261-274). Fondo FEN. Bogotá, Colombia.

Chamorro, C. (1990). Suelos. In J. Aguirre \& J. O. Rangel (Eds.), Biota y ecosistemas de Gorgona (pp. 65-72). Fondo FEN. Bogotá, Colombia.

Chao, A. (1984). Nonparametric estimation of the number of classes in a population. Scandinavian Journal of Statistics, 11: 265-270.

Chazdon R. L., Colwell, R. K., Denslow, J. S \& Guariguata, M. R. (1998). Statistical methods for estimating species richness of woody regeneration in primary and secondary rain forests of northeastern Costa Rica. In F. Dallmeier \& J. A. Comiskey (Eds.), Forest 
biodiversity research, monitoring and modeling: conceptual background and Old World case studies (pp. 285-309). Parthenon Publishing, Paris, France.

Colwell, R. K. (2009). EstimateS: Statistical estimation of species richness and shared species from samples. Version 8.2. User's Guide and Application. Retrieved from http://purl.oclc.org/estimates

Foggo, A., Ozanne, C. M. P., Spleight M. R. \& Hambler, C. (2001). Edge effects and tropical forest canopy invertebrates. Plant Ecology, 153: 347-359.

García-Aldrete, A. N. (1988). The psocids (Psocoptera) of Chamela, Jalisco, México. Species, diversity, abundance distribution and seasonal changes. Folia Entomológica Mexicana, 77: 63-84.

Gómez, A. (2009). Variación de la estructura y composición de los ensamblajes de macro invertebrados bénticos en la jerarquía espacial de los sistemas loticos del PNN Gorgona (Trabajo de Grado). Universidad de Antioquia, Medellín, Colombia.

González-Obando, R., García-Aldrete, A. N. \& Carrejo, N. S. (2011). A new species of Steleops Enderlein, and a Colombian record of $S$. pulcher New (Psocodea:'Psocoptera': Psocidae). Zootaxa, 2735: 23-27.

Hamada, N., Miyawaki, H. \& Yamada, A. (1995). Substrate specificity of epiphytic lichens. Annual Report of Osaka City Institute of Public Health Environmetal Science, 57:95-101.

Hammer, Ø., Harper, D. A. T. \& Ryan, P. D. (2001). PAST: Paleontological Statistics Software Package for Education and Data Analysis. Palaeontologia Electronica. Retrieved from http://palaeoelectronica.org/2001_1/ past/issue1_01.html).

Longo, M., Gómez, A., Blanco J. \& Zamora, Y. (2008). Cambios en dos escalas espaciales de la composición y estructura de la comunidad de insectos acuáticos en las quebradas perennes del PNN Gorgona (19912008). In Resúmenes VIII Seminario Colombiano de Limnología (pp. 145). Cali, Colombia.

Magurran, A. E. (1988). Ecological Diversity and its Measurement. Princeton University Press, Princeton, N. J.

Manzano, M. R., Nieser, N. \& Caicedo, G. (1995). Lista preliminar de hetéropteros acuáticos en la Isla Gorgona y Llanura del Pacífico. Biblioteca José Jeronimo Triana, 11:47-72.

Mockford, E. L. (1993). North American Psocoptera (Insecta). Flora and Fauna Handbook No. 10, Sandhill Crane Press, Gainesville, Florida.

Montoya-Lerma, J. \& Baena, M. (1995). Man-biting sand flies (Diptera: Psychodidae) from Gorgona, an island of the Pacific Coast of Colombia. Biblioteca José Jerónimo Triana, 11:73-78.

Palmer, M. W. (1990). The estimation of species richness by extrapolation. Ecology, 71: 1195-1198.
Pimienta, C., Montealegre, F., Chacón de Ulloa, P., Gonzalez, R. \& Mejía, J. (2008). Diversidad de artrópodos arborícolas en el PNN Gorgona (Cauca, Colombia). In Resúmenes XXXV Congreso de la Sociedad Colombiana de Entomología (pp. 47). Cali, Colombia.

Rangel, O. \& Rudas, A. (1990). Aspectos microclimáticos. In J. Aguirre \& O. Rangel (Eds.), Biota y Ecosistemas de Gorgona (pp. 41-51). Fondo FEN. Bogotá, Colombia.

Sarria, F., Montealegre, F., Mason, A. C. \& Pimienta, C. (2008). Saltamontes arbóreos del PNN Gorgona (Orthoptera: Tettigoniidae). In Resúmenes XXXV Congreso de la Sociedad Colombiana de Entomología (pp. 56). Cali, Colombia.

Simberloff, D. S. \& Wilson, E. O. (1969). Experimental Zoogeography of Islands: The Colonization of Empty Islands. Ecology, 50:278-296.

Smithers, C. N. (1990). Keys to the families and genera of Psocoptera (Arthropoda: Insecta). Technical Reports of the Australian Museum, 2: 1-82.

Statsoft, Inc. 2007. Statistica (data analysis software system), Version 8.0. Retrieved from: http://www. statsoft.com.

Thornton, I. W. B. (1985). The geographical and ecological distribution of arboreal Psocoptera. Annual Review of Entomology, 30:175-196.

Thornton, I. W. B., New, T. R. \& Vaughan, P. J. (1988). Colonization of the Krakatau Islands by Psocoptera (Insecta). Philosophical Transactions of the Royal Society Biological Sciences, 322: 427-443.

Torres, O., Pinilla, G. \& Chamorro, C. (1998). Artropofauna de los suelos de Isla Gorgona. Colombia Geográfica, 14(2):22-29.

Turner, B. D. \& Cheke, R. A. (1973). Two new species of Alaptus Westwood (Hymenoptera: Mymaridae) parasitising the eggs of Psocoptera. The Entomologist, 106: 279-283.

Turner, B. D. (1984). Psocoptera from Venezuela. A collection of psocids from the food boluses of swifts. Scandinavian Entomology, 15: 209-213.

UAESPNN. (2005). Plan de Manejo del Parque Nacional Natural Gorgona 2005-2009. Dirección Territorial Suroccidente. Gorgona, Colombia.

UASPNN. (2008). Línea Base PNN Gorgona. In C. L. Acevedo (Ed.), Implementación del subprograma manejo de vida silvestre en áreas del sistema de Parques Nacionales. Bogotá, Colombia.

Ulloa-Chacón, P. (1990). Biologie de la reproduction chez la petite fourmi de feu Wasmannia auropunctata (Roger) (Hymenoptera: Formicida) (Doctoral dissertation). Université de Lausanne, Switzerland.

Zapata, L., Beltrán, B., Collazos, A. \& Von Prahl, H. (1991). Estudio de la macrofauna asociada a la quebrada de La Camaronera, Isla Gorgona, Pacífico colombiano. Cespedesia, 18: 23-51. 